\title{
SISTEMA DE APOIO AO TURISMO ACESSÍVEL: ESTUDO E CONCEPTUALIZAÇÃO DE UMA APLICAÇÃO WEB
}

\author{
Pedro Teixeira ${ }^{1}$, Leonor Teixeira ${ }^{2}$ e Celeste Eusébio $^{3}$ \\ ${ }^{1}$ DEGEIT - University of Aveiro \\ ${ }^{2}$ IEETA / GOVCOPP / DEGEIT - University of Aveiro \\ ${ }^{3}$ GOVCOPP / DEGEIT - University of Aveiro \\ Campus Universitário de Santiago, 3810-193 Aveiro, Portugal
}

\begin{abstract}
RESUMO
As tecnologias de informação e da comunicação têm tido, cada vez mais, um papel crucial na área do turismo. Uma das áreas que mais pode vir a beneficiar das tecnologias é, claramente, a área do turismo acessível. No entanto, o turismo acessível, apesar de estar associado a um mercado com grande potencial, é ainda fortemente ignorado, pela existência de algumas barreiras. Soluções de base tecnológica podem ajudar a contornar alguns obstáculos, elevando o potencial deste mercado. Desta forma, o presente trabalho visa apresentar um estudo na área do turismo acessível, por forma a compreender os principais requisitos deste tipo de mercado e, consequentemente, conceptualizar uma solução Web, com o propósito de promover o turismo inclusivo. Para a conceptualização da solução adotou-se uma metodologia baseada na triangulação de métodos, seguindo-se a construção do modelo, com recurso à notação UML (Unified Modeling Language). Dada a importância deste tipo de soluções e dada a falta das mesmas no âmbito do turismo acessível, espera-se, com este estudo, contribuir com uma resposta positiva às necessidades levantadas no âmbito daquele mercado, ao mesmo tempo que se contribui com uma proposta de uma solução tecnológica que pode vir (i) a garantir as condições de acessibilidade na prática do turismo, por parte da procura, e (ii) a ajudar na promoção dos produtos e serviços acessíveis por parte da oferta.
\end{abstract}

\section{PALAVRAS-CHAVE}

Turismo Acessível, Aplicação Web, Modelo UML, Requisitos Funcionais, Requisitos Não-Funcionais

\section{INTRODUÇÃO}

O turismo é um setor em desenvolvimento, registando claros impactos económicos, tanto a nível nacional como internacional (Milicchio and Prosperi, 2016). O turismo acessível, tem acompanhado esta tendência, uma vez que se tem registado um aumento de turistas com algum tipo de necessidade ou incapacidade, situação que torna este mercado cada vez mais importante (Bekiaris et al., 2018). De entre os vários critérios de acessibilidade existentes para dar resposta a este mercado, tem-se, por um lado, a componente da acessibilidade física (infraestruturas), por outro, a componente da acessibilidade de comunicação que inclui, não só a disponibilização de informação sobre a acessibilidade, como também o nível de acessibilidade com que essa informação é comunicada. Para esta última componente, nomeadamente para garantir a acessibilidade na comunicação, releva-se o importante papel das tecnologias de informação e da comunicação (TICs) (Buhalis and Michopoulou, 2011). Na verdade, a era da digitalização a que atualmente se assiste tem promovido a criação de diferentes tipos de soluções tecnológicas, que, por sua vez, potenciam a criação de novos modelos de negócio ajustados a uma realidade mais digital. Na sua definição mais tradicional, e do ponto de vista organizacional, um Sistema de Informação (SI) pode ser definido como uma combinação de procedimentos, dados/informação, pessoas e tecnologias, que quando eficientemente combinados, potenciam o alcance dos objetivos definidos na estratégia de uma organização (Alter, 1992, p.7). Quando estes SI são usados para gerir e comunicar a informação de forma distribuída, usando para o efeito a rede da Internet, assumem a designação de SI baseados na Web (Yi and Hwang, 2003). 
O uso de SI, particularmente os suportados na Web, pode representar uma condição sine qua non para a promoção de condições de acesso à prática do turismo por parte de pessoas com algum tipo de necessidade especial ou incapacidade, na medida em facilitam o acesso a diferentes atividades turísticas, combatendo assim a exclusão social. Este tipo de soluções tecnológicas, não só potenciam experiências turísticas a qualquer pessoa, independentemente do seu grau de necessidade ou incapacidade, como asseguram um maior valor ao cliente, ajudando na sua integração social.

Apesar dos benefícios que advém do uso deste tipo de soluções tecnológicas, atualmente ainda se regista uma evidente falta de plataformas orientadas à promoção do turismo acessível. Como tal, é objetivo deste trabalho apresentar um estudo que culmine na proposta de um modelo de uma plataforma Web (a que se deu o nome de AccessTour@WebApp) capaz de mediar as necessidades de informação entre a procura e a oferta, no âmbito do turismo acessível.

\section{REVISÃO DA LITERATURA}

\subsection{Turismo Acessível}

O Turismo é uma atividade económica e social de grande relevância (Turner, 2017). De notar que o desenvolvimento desta atividade não só proporciona benefícios para os seus praticantes, como também para os agentes económicos que integram este setor. No entanto, existem ainda muitos grupos na nossa sociedade que não conseguem participar de forma plena em atividades turísticas, devido a diversas barreiras (Buhalis and Darcy, 2011, p.50). Neste sentido, alguns autores que trabalham nesta área do conhecimento têm recomendado a criação de condições que promovam a acessibilidade dos destinos turísticos, por forma a ajudar a proporcionar experiências turísticas a todas as pessoas, independentemente do seu grau de incapacidade ou necessidade (Michopoulou et al., 2015). Assim, o desenvolvimento do turismo acessível torna-se indispensável para a inclusão de pessoas com incapacidades na sociedade, sendo necessário o envolvimento de todos os stakeholders do sistema turístico, no sentido de disponibilizar produtos e serviços turísticos adequados, adaptados e acessíveis.

O mercado de turismo acessível inclui pessoas com diferentes necessidades a nível físico e de comunicação (Michopoulou and Buhalis, 2013), mais propriamente pessoas com incapacidades físicas, mentais e intelectuais ou com incapacidades a nível dos diferentes sentidos, dificultando a sua participação ativa na sociedade (United Nations, 2006, p.4). A percentagem de turistas com incapacidades representa já um segmento de mercado muito importante, sendo que o desenvolvimento deste tipo de turismo depende fortemente da criação de condições para que seja possível um turismo inclusivo. De facto, esta necessidade potência o aparecimento de novas oportunidades de negócios por parte das diferentes entidades que operam na cadeia do turismo, nos quais se incluem, por exemplo, as unidades de alojamento, as empresas de lazer, os operadores turísticos, entre outros. Outro importante especto deste tipo mercado, é a capacidade de reduzir a sazonalidade verificada no turismo de uma forma geral, já que pessoas com incapacidades (PCI) normalmente consideram a prática do turismo em períodos de época baixa (Ribeiro et al., 2018). Na verdade, alguns estudos estimam que até 2020, cerca de $25 \%$ dos gastos com viagens e lazer serão provenientes de turistas com incapacidades (Bekiaris et al., 2018). Mas apesar desta tendência, este mercado é ainda fortemente ignorado por parte da oferta turística (Portales, 2015).

$\mathrm{Na}$ verdade, o turismo acessível tem vindo a ganhar algum destaque nos últimos tempos, no entanto, ainda existem algumas barreiras que impedem a realização deste tipo de turismo, em pleno. Alguns estudos chegam mesmo a evidenciar que, se as condições de acessibilidade estivessem asseguradas, as pessoas viajariam mais (Pühretmair, 2004). Pois as barreiras existentes à prática do turismo por parte deste público criam "obstáculos", ameaçando a qualidade de vida das pessoas e a sua independência (Popiel, 2016). De entre as várias barreiras, e tal como já foi referido, realça-se a falta de informação sobre a acessibilidade, bem como a falta de informação acessível (Stumbo and Pegg, 2008). A informação sobre a acessibilidade permite perceber se os requisitos do turista serão tidos em conta ao longo da viagem e durante as experiências turísticas, bem como se estão reunidas todas as condições para a realização do turismo, sem qualquer constrangimento. Já a acessibilidade da informação permite garantir que toda a informação está acessível, ou seja, chega à pessoa que dela necessita, sem nenhuma perda de valor. É precisamente na relação de estes dois aspetos, i.e., informação sobre acessibilidade e acessibilidade da informação, que entra o papel das TICs, assumindo importância primordial, enquanto ferramentas promotoras do turismo acessível. 


\subsection{As TICs no Turismo Acessível}

A inovação tecnológica tornou possível o desenvolvimento nos mais diferentes tipos de indústria (Sedlar et al. 2018). Neste contexto, entende-se por tecnologia qualquer dispositivo, componente de networking, aplicação ou sistema que, quando contextualizados de forma sistémica, permitem a interação com o mundo digital (Rouse, 2014). Dentro destas diversas tecnologias, surgem as TICs, responsáveis por garantir que a informação e o conhecimento sejam divulgados e comunicados, tendo por base a emissão e transmissão de dados digitais.

No caso do turismo acessível, as TICs poderão assumir um papel importante na medida em que podem ser responsáveis pela divulgação de informação, acerca das condições de acessibilidade das diferentes atividades e/ou produtos turísticas (Buhalis and Michopoulou, 2011). Na mesma linha de pensamento, com a Web a representar um pilar fundamental na sociedade, os sistemas de informação baseados na Web representam as fontes de informação preferenciais dos turistas e, como tal, fundamentais na promoção de um turismo mais acessível (Rumetshofer and Woss, 2005). Este tipo de soluções tecnológicas é capaz de fornecer uma base de conhecimento partilhado e adaptável a qualquer tipo de necessidade e/ou incapacidade, sendo, portanto, adequado à promoção do turismo acessível. De facto, com as TICs é possível servir diferentes necessidades de informação, necessidades essas que podem ser provenientes de diferentes tipos de incapacidade.

De realçar que as TICs não servem apenas as necessidades de informação no turismo acessível, mas podem ser úteis noutros contextos de acessibilidade. Por exemplo, tecnologias relacionadas com sistemas de localização são essenciais na construção de bases de dados geográficas acessíveis (Barbeau et al., 2010). Também as aplicações móveis podem apresentar mecanismos proactivos e características colaborativas (Emrouzeh et al., 2017). Em suma, as TICs focam-se, não só na componente tecnológica de divulgação da informação, mas também em mecanismos que permitam assegurar a conexão com o mundo digital, nomeadamente tecnologias de assistência e/ou tecnologias adaptativas (Fall et al., 2018). Alguns exemplos práticos da aplicação das TICs no turismo acessível estão relacionados com a assistência na visita a museus como mostram o estudos de Angkananon et al., (2015), Haworth and Williams (2012) e Mesquita and Carneiro, (2016).

Sendo as aplicações Web, plataformas ideais para divulgação de informação, e sendo a informação um aspeto crítico para o turismo, justifica-se, claramente, a sua utilização na promoção do turismo para todos, já que, como em qualquer outro domínio, a informação é a chave de sucesso para elevar qualquer negócio.

\section{APLICAÇÃO WEB PARA O TURISMO ACESSÍVEL}

\subsection{Objetivo da Aplicação}

Como já foi referido, a falta de informação sobre a acessibilidade e a falta de informação acessível representam as principais barreiras no turismo acessível, existindo, portanto, a necessidade de se criarem soluções que permitam ultrapassar estes embargos. De facto, quanto maiores os requisitos de acessibilidade, maior também será a necessidade de informação detalhada sobre esses requisitos (Bowtell, 2015).

Este estudo, que faz parte de um projeto mais alargado, pretende apresentar soluções para os problemas advindos do contexto anteriormente traçado, propondo um modelo de uma plataforma Web (a que se deu o nome de AccessTour@WebApp) capaz de mediar as necessidades de informação entre a procura e a oferta, no âmbito do turismo acessível. A importância deste estudo é relevada pela falta de plataformas com respostas específicas àquelas necessidades, mais concretamente que permitam uma integração e interação entre os diversos stakeholders envolvidos na cadeia de valor, com fim à promoção das condições de acessibilidade dos destinos turísticos. A AccessTour@WebApp visa, assim, facilitar a comunicação e partilha de informação entre turistas, designadamente PCI e organizações turísticas. Espera-se que uma solução desta natureza possa ajudar na promoção do mercado do turismo acessível, na medida em que: (i) possibilita o acesso às PCI, de forma acessível, à informação sobre a acessibilidade dos destinos, o que lhes permite encontrar soluções turísticas adaptadas às suas necessidades e/ou incapacidades; e (ii) representa uma excelente oportunidade de interagir com um mercado em ascensão, alcançando potenciais clientes, através da divulgação das ofertas de turismo acessível, numa plataforma dedicada para o efeito. 


\subsection{Metodologia}

De forma a perceber o tipo de informação e principais funcionalidades a contemplar na solução a propor, foi conduzido um estudo de levamento de requisitos, suportado em três abordagens metodológicas diferentes: (i) revisão da literatura; (ii) análise da acessibilidade de websites pertencentes a hotéis localizados na zona centro de Portugal e (iii) análise de conteúdo de plataformas concorrentes. O primeiro método aferiu e analisou conceitos já existentes na literatura, relacionando os SI com o potencial que os mesmos poderiam promover naquele mercado. O segundo método de pesquisa foi um estudo prático, onde se analisou o nível de acessibilidade de websites de diferentes hotéis, utilizando para isso duas ferramentas de análise automática (AccessMonitor e TAW). Este estudo prático permitiu perceber quais os principais problemas de acessibilidade relacionados com a arquitetura dos websites, e que aspetos fundamentais se encontravam em falta. Apesar dos resultados deste trabalho não serem o foco deste estudo, os mesmos contribuíram para identificar um conjunto de requisitos não funcionais que deverão estar presentes nas aplicações tecnológicas que servem este mercado. O terceiro método utilizado analisou algumas plataformas nacionais e internacionais, ao nível de conteúdos e funcionalidades, que servem parcialmente este tipo de mercado.

Os requisitos identificados foram classificados em dois tipos: requisitos funcionais e não-funcionais. Os requisitos funcionais são aqueles que determinam o que o sistema deve fazer, enquanto que os não-funcionais descrevem a qualidade com que os requisitos funcionais devem ser oferecidos (Zhou et al., 2004). Nestes últimos, incluem-se os requisitos de comunicação, que no presente estudo se prendem com os requisitos de acessibilidade.

Das três fontes de dados utilizadas, resultou uma matriz de triangulação de dados, composta pela lista de requisitos, bem como pelo método que originou cada requisito. A importância desta matriz não só está nas duas dimensões acima citadas (requisito x método), mas principalmente no grau de importância que se pode extrair de cada requisito (Teixeira et al., 2012). Depois de concluída a fase de recolha de requisitos, foi elaborado o modelo do AccessTour@WebApp, usando, para o efeito, os diagramas de use cases e de classes da notação UML (Unified Modeling Language). O primeiro diagrama - use-cases - permitiu representar os diferentes atores bem como as interações destes com o sistema. O segundo diagrama - classes -, possibilitou perceber o modelo abstrato de dados que está na base do domínio do problema.

\subsection{Conceptualização da AccessTour@WebApp}

O modelo proposto será apresentado nesta secção com recurso a sois diagramas da UML: diagrama de use cases (ver Figura 1) e o diagrama de classes (ver Figura 2).

O diagrama de use-cases, representado na figura 1 identifica as diferentes interações existentes entre utilizadores e sistema. O objetivo principal do diagrama é demonstrar os diferentes serviços presentes na aplicação Web. Os Atores representam os perfis de utilizadores que interagem com o sistema, identificando-se neste projeto atores do lado da procura (Visitante e Visitante Registado) e atores do lado da oferta (Organização Turística e Organização Turística Membro). Existe ainda um Ator (Administrador) responsável pela gestão do sistema, nomeadamente em termos de serviço de manutenção dos dados. Cada ator tem associado um conjunto de serviços (use-cases), tal como se pode observar na figura 1.

O Visitante acede a diversos tipos de conteúdo no sistema, podendo pesquisar por ofertas de turismo acessível e personalizar as definições, de acordo com as suas necessidades. Por exemplo, turistas daltónicos podem modificar as cores do sistema, e pessoas com visão reduzida podem ativar o controlo por voz. Existe ainda a possibilidade de um Visitante se registar na plataforma, tornando-se um Visitante Registado. Após o registo ser concluído, os turistas terão acesso a áreas restritas do sistema, a título de exemplo, espaço de partilha, com outros utilizadores registados, de fotos, vídeos e outros elementos, podendo estes elementos estarem relacionados com as experiências turísticas. O Visitante Registado poderá também avaliar e dar feedback de ofertas turísticas que tenha experienciado, de forma a melhorar as condições de acessibilidade para outros utilizadores.

A Organização Turística terá acesso a um conjunto de informação promocional, sendo que a funcionalidade de destaque para este ator é a do registo e visualização e concordância das condições de acesso. Por razões de segurança e cumprimento de critérios, existe a necessidade de fazer obrigatoriamente um pré-registo na plataforma, para poder usufruir de um conjunto de funcionalidades restritas que só as Organização Turística Membro terão acesso. Após o registo, e após a validação do registo pelo 
Administrador, aquele assumirá, então, o papel de Organização Turística Membro. Este ator será responsável por inserir no sistema ofertas de turismo acessível e disponibilizar também toda a informação, relativamente à acessibilidade (física e de comunicação) da(s) sua(s) oferta(s). Estas ofertas poderão ser alteradas ou modificadas, e se for possível deverão vir acompanhadas de um relatório, contendo informação mais detalhada, acerca das condições de acessibilidade.

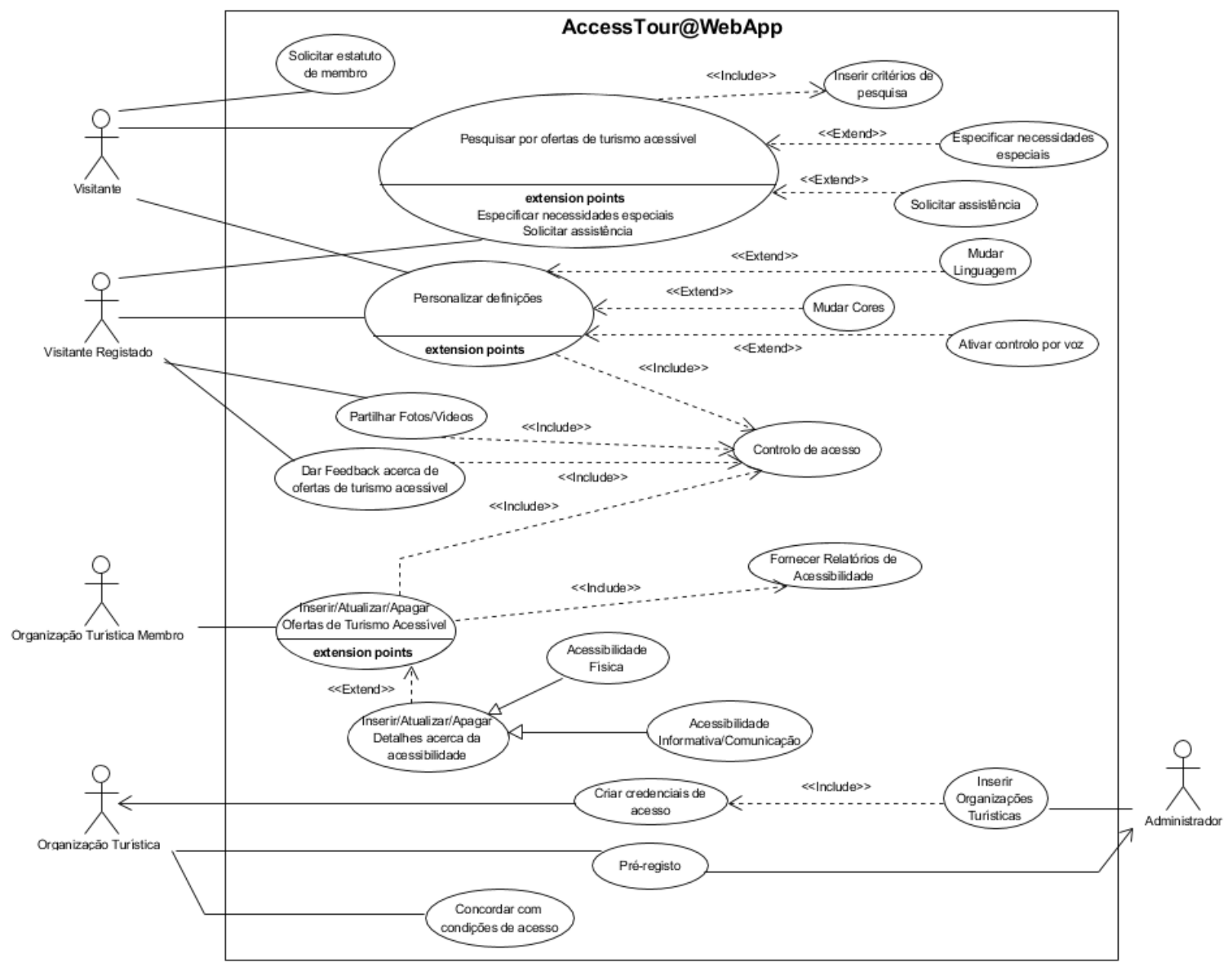

Figura 1. Diagrama de Use-cases do AccessTour@WebApp

O diagrama de classes representado na figura 2 apresenta uma descrição de alto nível das principais classes, atributos e relacionamentos que compõem o modelo do AccessTour@WebApp.

Como é possível ver no diagrama da figura 2, diferentes tipos de ofertas poderão ser inseridos na aplicação Web, nomeadamente: Alojamento (hotéis, hostels e pousadas), Restauração (restaurantes, pastelarias e coffee shops), Transportes, Agências de Viagens e Operadores Turísticos (AVOP) e, ainda, Atividades Culturais, Recreativas e Desportivas (ACRD), como são exemplo os cinemas, teatros, museus e galerias de arte. Para além das ofertas, é necessário registar informação acerca da acessibilidade física e de comunicação, associada às diversas ofertas.

Para um Visitante usufruir em pleno do AccessTour@WebApp é especialmente relevante registar-se e, consequentemente, indicar o tipo de incapacidade que possui, de forma a que o sistema seja capaz de recomendar as ofertas turísticas mais apropriadas para aquele turista. Pois pretende-se que o sistema apresente ofertas em tempo real ao turista, tendo por base o seu tipo de necessidade ou incapacidade, previamente registada. De salientar que este tipo de funcionalidade está igualmente dependente da informação introduzida pelo turista aquando da viagem, i.e., do registo do período da visita, podendo o sistema notificar, através do envio de mensagens (áudio e texto) para o contacto do Visitante Registado ou para o contacto do cuidador que acompanha o turista, sobre ofertas de interesse nas proximidades do local da visita. Um exemplo prático poderá ser aquando de uma visita a uma qualquer atração ou destino, a AccessTour@WebApp poderá dar informação ao turista acerca de lugares de estacionamento para pessoas 
com mobilidade reduzida, ou indicar se existem intérpretes ou guias com conhecimento de linguagem gestual.

Em termos de requisitos não funcionais, pretende-se que este sistema permita o acesso a qualquer tipo de utilizador, independentemente da sua condição.

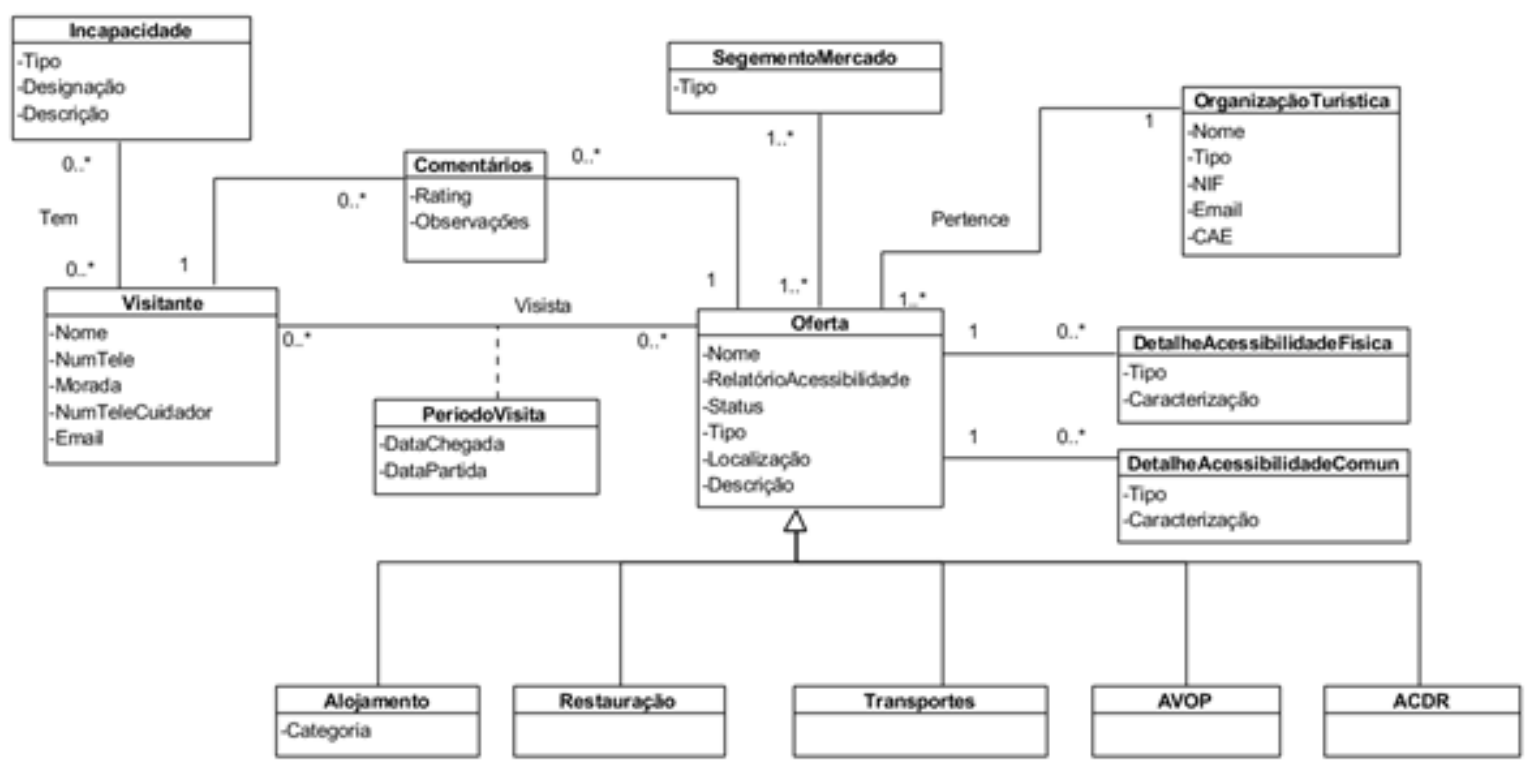

Figura 2. Diagrama de Classes do AccessTour@WebApp

\section{CONCLUSÕES E TRABALHO FUTURO}

O mundo tecnológico está a revolucionar o turismo. Um dos setores que mais pode beneficiar de novas TICs é, sem dúvida, o do turismo. Neste sentido, as aplicações Web podem ter um papel importante na promoção e desenvolvimento deste tipo de setor, com grande impacto no turismo acessível. No entanto, soluções deste género, que sirvam este tipo de mercado específico, são ainda muito limitadas. Dada a importância deste tipo de soluções e a necessidade de aplicações práticas neste tipo de mercado, foi objetivo deste trabalho apresentar um estudo que culminou na proposta de uma solução tecnológica para a promoção de produtos e serviços no âmbito do turismo acessível.

O processo de compreensão e levantamento de requisitos baseou-se numa abordagem de triangulação de métodos, sendo os requisitos oriundos de um, ou mais, dos métodos usados: (i) revisão da literatura; (ii) análise da acessibilidade de websites pertencentes a hotéis localizados na zona centro de Portugal e (iii) análise de conteúdo de plataformas concorrentes. Desta abordagem resultou uma matriz de triangulação com uma lista de requisitos funcionais e não-funcionais, que deram forma à solução e conduziram à definição do modelo proposto e concretizado com base nos diagramas de use-cases e de classes da notação UML.

Com este estudo, espera-se assim contribuir com uma proposta de solução tecnológica que promova canais e formas de comunicação eficazes entre a procura (i.e., PCI) e a oferta turística, garantindo, portanto, as condições de acessibilidade por parte das PCI, bem como a divulgação dos serviços, por parte da oferta, junto a um mercado potencialmente lucrativo.

Em termos de trabalho futuro, espera-se vir a dar continuidade a este projeto, nomeadamente aferir a viabilidade e fiabilidade do sistema junto dos stakeholders, nomeadamente turistas que sofram com algum tipo de incapacidade, bem como com organizações turísticas que desejem divulgar ofertas de turismo acessível. Sendo o turista com incapacidades, o principal elemento deste serviço, deverá ser complementada a análise de requisitos, junto destes. Neste sentido, esta averiguação será feita através da resposta a questionários, podendo ser identificados novos requisitos. Uma vez que as organizações turísticas são também uma parte fundamental, o levantamento de requisitos junto destas entidades será também conduzido com base em questionários. Posteriormente espera-se avançar com o desenvolvimento de um protótipo 
funcional da plataforma, seguindo uma abordagem iterativa e incremental, bem como conduzir um conjunto de testes de aceitação, por forma a compreender a relevância das funcionalidades e informação presentes no sistema, bem como identificar novas funcionalidades, caso venham a surgir novas necessidades.

\section{AGRADECIMENTO}

Este trabalho foi desenvolvido no âmbito do projeto de investigação ACTION - POCI-01-01-0145-FEDER030376 -, financiado pelo FEDER, através do COMPETE2020 - Programa Operacional Competitividade e Internacionalização (POCI), e por fundos nacionais (OE), através da FCT/MCTES.

\section{REFERÊNCIAS}

Alter, S. 1992, Information Systems: A Management Perspective, Addison-Wesley,USA

Angkananon, K., Wald, M. and Gilbert, L. 2015, 'Technology enhanced interaction framework and method for accessibility in Thai museums', 3rd International Conference on Information and Communication Technology (ICoICT), IEEE, Bali, Indonesia, pp. 316-321.

Barbeau, S., Georggi, N. and Winters, P. 2010, 'Global positioning system integrated with personalized real-time transit information from automatic vehicle location', Transportation Research Record: Journal of the Transportation Research Board, vol. 2143, no. 1, pp. 168-176.

Bekiaris E; Loukea M; Spanidis, P; Ewing.S; Denninghaus, M; Ambrose, I; Papamichail, K. and Castiglioni, R.C. 2018, Research for TRAN Committee - Transport and tourism for persons with disabilities and persons with reduced mobility.

Bowtell, J. 2015, 'Assessing the value and market attractiveness of the accessible tourism industry in Europe: a focus on major travel and leisure companies', Journal of Tourism Futures, vol. 1, no. 3, pp. 203-222.

Buhalis, D. and Darcy, S. 2011, Accessible Tourism Concepts and Issues. Channel View Publications, UK

Buhalis, D. and Michopoulou, E. 2011, 'Information-enabled tourism destination marketing: addressing the accessibility market', Current Issues in Tourism, vol. 14, no. 2, pp. 145-168.

Buhalis, D. and Michopoulou, E. 2011, 'Information-enabled tourism destination marketing: Addressing the accessibility market', Current Issues in Tourism, vol. 14, no. 2, pp. 145-168.

Emrouzeh, M.P., Dewar, K., Fleet, G. and Bourgeois, Y. 2017, 'Implementing ICT for Tourists with Disabilities', Proceedings of the 2017 International Conference on E-Education, E-Business and E-Technology, no. 6, pp. 50-53.

Fall, C.L., Quevillon, F., Blouin, M., Latour, S., Campeau-Lecours, A., Gosselin, C. and Gosselin, B. 2018, 'A Multimodal Adaptive Wireless Control Interface for People with Upper-Body Disabilities', IEEE Transactions on Biomedical Circuits and Systems, vol. 12, no. 3, pp. 564-575.

Haworth, A. and Williams, P. 2012, 'Using QR codes to aid accessibility in a museum', Journal of Assistive Technologies, vol. 6, no. 4, p. 285-291.

Mesquita, S. and Carneiro, M. 2016, 'Accessibility of european museums to visitors with visual impairments', Disability and Society, vol. 31, no. 3, pp. 373-388.

Michopoulou, E. and Buhalis, D. 2013, 'Information provision for challenging markets: The case of the accessibility requiring market in the context of tourism', Information and Management, vol. 50, no. 5, pp. 229-239.

Michopoulou, E., Darcy, S., Ambrose, I. and Buhalis, D. 2015, 'Accessible tourism futures: the world we dream to live in and the opportunities we hope to have', Journal of Tourism Futures, vol. 1, no. 3, pp. 179-188.

Milicchio, F. and Prosperi, M. 2016, 'Accessible tourism for the deaf via mobile apps', 9th ACM International Conference on PErvasive Technologies Related to Assistive Environments - PETRA '16, ACM Press, New York, New York, USA, pp. 1-7.

Popiel, M. 2016, Barriers in Undertaking Tourist Activity by Disabled People, no. 3, pp. 103-110.

Portales, R.C. 2015, 'Removing “invisible” barriers: opening paths towards the future of accessible tourism', Journal of Tourism Futures, vol. 1, no. 3, pp. 269-284.

Pühretmair, F. 2004, 'It's time to make eTourism accessible', Computers helping people with special needs, pp. $272-279$.

Ribeiro, F., Silva, A., Barbosa, F., Silva, A. and Metrôlho, J. 2018, 'Mobile applications for accessible tourism: overview, challenges and a proposed platform', Information Technology and Tourism, vol. 19, no. 1-4, pp. $29-59$. 
Rouse, M. 2014, 'ICT (information and communications technology, or technologies)', Tech Target, Available at <https://searchcio.techtarget.com/definition/ICT-information-and-communications-technology-or-technologies> (Accessed 23 January 2019).

Rumetshofer, H. and Woss, W. 2005, 'Semantic maps and meta-data enhancing e-accessibility in tourism information systems', 16th International Workshop on Database and Expert Systems Applications (DEXA'05), IEEE, pp. 881-885.

Sedlar, U., Kos, A., Pustisek, M., Bester, J., Pogacnik, M., Mali, L. and Stojmenova Duh, E. 2018, 'Tackling the Challenges of ICT Innovation and Talents for Industry 4.0', Ipsi Bgd Transactions on Internet Research, vol. 14, no. 1.

Stumbo, N.J., Pegg, S., 2008. Travelers and tourists with disabilities: a matter of priorities and loyalties. Tourism Review International 8, 195-209.

Teixeira, L., Ferreira, C. and Santos, B.S. 2012, 'User-centered requirements engineering in health information systems: A study in the hemophilia field', Computer Methods and Programs in Biomedicine, vol. 106, no. 3, pp. 160-74.

Turner, R. 2017, The Economic Impact of Travel.Available at <https://www.wttc.org/-/media/files/reports/economicimpact-research/regions-2017/world2017.pdf> (Accessed 25 May 2019).

United Nations 2006, The UN convention on the rights of persons with disabilities, Available at <https://www.un.org/disabilities/documents/convention/convoptprot-e.pdf > (Accessed 10 April 2019).

Yi, M.Y. and Hwang, Y. 2003, 'Predicting the use of web-based information systems: Self-efficacy, enjoyment, learning goal orientation, and the technology acceptance model', International Journal of Human Computer Studies.

Zhou, Y.J., Hyvonen, S.L. and Louise, S. 2004, Functional Requirements and Non-Functional Requirements: A Survey, Dissertation. 